\title{
The prognostic significance of tumor lymphangiogenesis and lymphatic vessel density in endometrioid carcinoma of the uterine corpus
}

\author{
MIKA SAKAKI $^{1}$, MASANORI YASUDA ${ }^{1}$, MITSUTAKE YANO ${ }^{1}$, YOSHIYA GOTO ${ }^{1}$, \\ MASARU NAKAMURA ${ }^{1}$, HIDEKAZU KAYANO ${ }^{2}$ and KOSEI HASEGAWA ${ }^{3}$ \\ ${ }^{1}$ Department of Pathology, Saitama Medical University International Medical Center; \\ ${ }^{2}$ Faculty of Health Sciences, Saitama Medical University; ${ }^{3}$ Department of Gynecologic Oncology, \\ Saitama Medical University International Medical Center, Hidaka, Saitama 350-1298, Japan
}

Received February 13, 2017; Accepted August 23, 2017

DOI: $10.3892 / \mathrm{ol} .2017 .6885$

\begin{abstract}
The present study aimed to assess the prognostic significance of tumor lymphangiogenesis and lymphatic vessel density (LVD) in endometrioid carcinoma of the uterine corpus. The association between LVD and various factors, including lymphatic vessel invasion (LVI), and nodal metastases, were investigated. Among 202 surgically treated cases, 19 cases with nodal metastases with the infiltration reaching the outer-half myometrium $\left(\mathrm{N}^{+}\right.$group) were selected. The LVDs of hotspots in each case were examined at 100x magnification on D2-40 stained slides of the inner- and outer-half myometrium of the peritumoral, and control compartments. Furthermore, for the peritumoral compartment, the LVDs in two types of areas, LVI-present and LVI-absent, were examined for each location of myometrium, which amounted to 6 spots/case. They were statistically compared. Second, to determine whether the existing myometrium influenced LVD, LVDs in the intratumoral compartment were investigated, which were defined as a region where residual muscular tissue was unrecognizable with desmin-immnostaining. As a negative control group, LVDs of the inner- and outer-half myometrium of the peritumoral, and control compartments of 29 cases without nodal metastases in stage IB ( $\mathrm{N}^{-}$group) were examined. No significant association was identified between the LVD and frequency of LVI. However, the LVDs in the peritumoral compartment and inner-half myometrium were higher compared those in the control compartment, and outer-half myometrium in $\mathrm{N}^{+}$and $\mathrm{N}^{-}$groups, respectively. No
\end{abstract}

Correspondence to: Dr Mika Sakaki, Department of Pathology, Saitama Medical University International Medical Center, 1397-1 Yamane, Hidaka, Saitama 350-1298, Japan

E-mail: amjy106@ybb.ne.jp

Key words: tumor lymphangiognesis, lymphatic vessel density, lymphatic vessel invasion, no correlation, other influence on lymphatic vessel density significant differences were identified in LVD of the peritumoral compartment between groups. The lymphatic vessels were absent where the existing muscular tissue was absent in $16 / 19$ cases. Although higher LVDs in the peritumoral compartment suggested tumor lymphangiogenesis, it was not associated with LVI and nodal metastases. Other factors that influenced LVD were the location in the myometrium and the existing myometrium.

\section{Introduction}

Increased lymphatic vessel density (LVD) in the peritumoral and/or intratumoral regions has been reported to be associated with the presence of lymphatic vessel invasion (LVI), resulting in regional lymph node metastases and poor prognosis, and reflects tumor lymphangiogenesis in carcinomas of various sites (1-7).

Endometrioid carcinoma is the most frequently-occurring carcinoma of the uterine corpus. The incidence of nodal metastases is relatively low and the presence or degree of lymphovascular invasion was related to the nodalmetastases or worse survival (8-11). However, an association between the LVD and the frequency of LVI has not been reported. Histologically, the lymphatic vessels with tumor invasion are usually scattered, not continuous or numerous in the peritumoral region of the cases even with nodal metastases. In the articles published so far regarding the carcinomas of other sites (1-7), the association has been examined by comparing the LVDs between two groups, one with the presence of LVI and the other with the absence of LVI. We think that if tumor lymphangiogenesis causes LVI by increasing LVD, the LVD in an area including LVI should be higher than in an area not including LVI within a case. So we would select cases with nodal metastases and investigate the association in a different, more detailed way, by examining the LVDs in two types of hot spots, an LVI-present $\left(\mathrm{LVI}^{+}\right)$area and an LVI-absent $\left(\mathrm{LVI}^{-}\right)$area, in both the inner- and outer-half myometrium of the peritumoral region in a case. Since the distribution of lymphatic vessels in the uterine corpus tends to be irregular and random $(12,13)$, the numbers of lymphatic vessels could originally be different between the inner- and the outer-half 
myometrium. We would also investigate the LVDs in the control regionfor the comparison.

Second, we would examine whether the destruction of the existing myometrium influences the LVD, by investigating the LVD in the intratumoral region, which we define as a region where residual muscular tissue is not recognizable with desmin immnostaining. In the breast and prostate (14-16), the LVDs have been reported to increase in the peritumoral region but not in the intratumoral region, where the existing architecture is destroyed by carcinoma.

In addition, we would investigate the LVDs in inner- and outer-half myometrium of the peritumoral and control regions in cases without nodal metastases as a negative control group.

By clarifying the questions mentioned above, we would assess the prognostic significance of tumor lymphangiogenesis and LVD in endometrioid carcinoma of the uterine corpus.

\section{Materials and methods}

Case selection. Out of the 222 cases diagnosed as endometrioid carcinoma of the uterine corpus that underwent total abdominal hysterectomy, bilateral salpingo-oophorectomy and lymph node dissection (pelvic or pelvic and para-aortic lymph nodes) without chemoradiation therapy at our institution between 2008 and 2015, 20 cases which also had endometrioid carcinoma in the ovary were excluded. Twenty-four cases of the remaining 202 cases showed nodal metastases. Nineteen of these 24 cases, where the carcinoma reached the outer-half myometrium $\left(\mathrm{N}^{+}\right.$group), were selected. As a negative control group, 29 cases in stage IB with more than 20 lymph nodes dissected showing no metastases $\left(\mathrm{N}^{-}\right.$group), were selected during the same period.

The age of the $\mathrm{N}^{+}$group ranged from 38 to 74 with a mean of 58.4. The tumor grade was assessed according to the FIGO system, and the cases consisted of $\mathrm{G} 1, \mathrm{n}=7, \mathrm{G} 2, \mathrm{n}=8$ and G3, $\mathrm{n}=4$. The age of the $\mathrm{N}^{-}$group ranged from 39 to 73 with a mean of 58.6. The cases consisted of G1, n=16, G2, $n=8$ and $\mathrm{G} 3, \mathrm{n}=5$.

Immunohistochemistry. Several paraffin blocks, containing lesions of the deepest invasion, LVI and control areas for each case, were chosen for immunohistochemical examination using antibodies against D2-40 (M3619, Dako, Glostrup, Denmark) and desmin (D33; Dako). A D2-40 antibody was used for the detection of lymphatic vessels and a desmin antibody for the recognition of the existing muscular tissue. Four-micrometer-thick sections were obtained from paraffin-embedded blocks. Immunohistochemistry was conducted using a BenchMark XT (Ventana Medical Systems Inc., Tucson, AZ, USA) and an I-View DAB detection kit (Ventana Medical Systems Inc.) at a dilution of 1:100 for both antibodies.

Quantification of lymphatic vessels. The three compartments, the intratumoral, peritumoral and control compartments of the uterine corpus were defined as follows. The intratumoral compartment was defined as the area encompassing all the cancer glands. The stroma of the intratumoral compartment was the region where the residual muscular tissue was not recognizable with desmin-immunostaining. The peritumoral compartment was a $2 \mathrm{~mm}$-wide area around the intraumoral

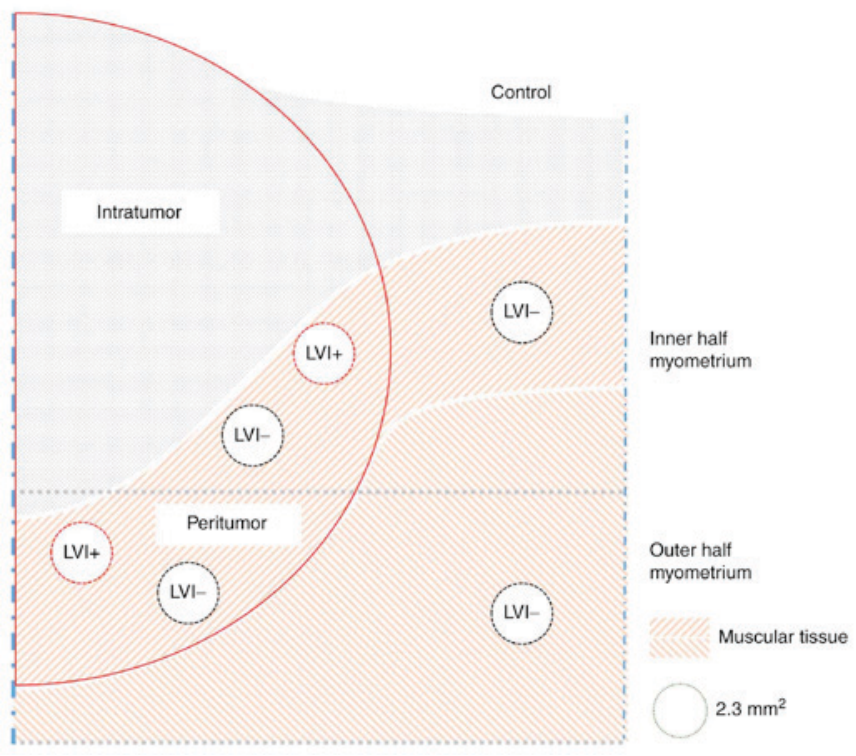

Figure 1. LVDs of 6 hot spots examined. Six circular areas surrounded by dotted lines showed $\mathrm{LVI}^{+}$and $\mathrm{LVI}^{-}$areas of peritumoral compartment, and $\mathrm{LVI}^{-}$areas of control compartment in inner- and outer-half myometrium. LVD, lymphatic vessel density; LVI, lymphatic vessel invasion.

compartment. The peritumoral compartment was defined as a region where the existing muscular tissue was preserved. The control compartment was an area beyond the peritumoral compartment.

A hot spot on a D2-40 immunostained slide was chosen at 100x magnification through cell Sense Standard (a software installed to the camera connected to the microscope, Olympus) and the view of the chosen area $\left(2.3 \mathrm{~mm}^{2}\right)$ was printed out for manual counting of lymphatic vessels. We refer to the number of the lymphatic vessels per $2.3 \mathrm{~mm}^{2}$ as LVD. Hot spots were chosen for the $\mathrm{LVI}^{+}$and $\mathrm{LVI}^{-}$areas of both the inner- and outer-half myometrium in the peritumoral compartment, and similarly for the $\mathrm{LVI}^{-}$area in the control compartment, which amounted to 6 spots examined per case (Fig. 1). In addition, for the $\mathrm{LVI}^{+}$area, the number of lymphatic vessels with tumor invasion (LVD with tumor invasion) was also counted.

For the intratumoral region, when the lymphatic vessels were recognized, the LVD was similarly counted and if LVI was present, the LVD with tumor invasion was also counted.

For the $\mathrm{N}^{-}$group, the LVDs in both inner- and outer-half myometrium of the peritumoral and control compartments were counted. Only one hot spot was examined in each part of the myometrium of the peritumoral compartment regardless of LVI.

Statistical analysis. The LVDs in the peritumoral and control compartments were statistically compared within each group. And the LVDs in the inner- and outer-half myometrium of the peritumoral regions were compared respectively betweengroups, in which case the higher LVD of each location of the peritumoral compartment in the $\mathrm{N}^{+}$group was chosen for the comparison regardless of the LVI status. Statistical analyses were performed using Statcel 3. Student's t-test, Welch t-test or Mann-Whitney's test was used for the comparison. $\mathrm{P}<0.05$ was considered to indicate a statistically significant difference. 
Table I. Location of LVI in the peritumoral compartment of 19 cases in $\mathrm{N}^{+}$group.

\begin{tabular}{lccccc}
\hline Location & \multicolumn{5}{c}{ Presence of LVI } \\
$\begin{array}{l}\text { Inner-half } \\
\text { myometrium }\end{array}$ & + & + & - & - & \\
$\begin{array}{l}\text { Outer-half } \\
\text { myometrium }\end{array}$ & + & - & + & - & \\
\begin{tabular}{l} 
Number of cases \\
\hline
\end{tabular} & 14 & 1 & 3 & $1^{\text {a }}$ & 19 \\
\hline
\end{tabular}

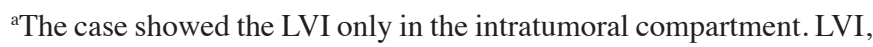
lymphatic vessel invasion.

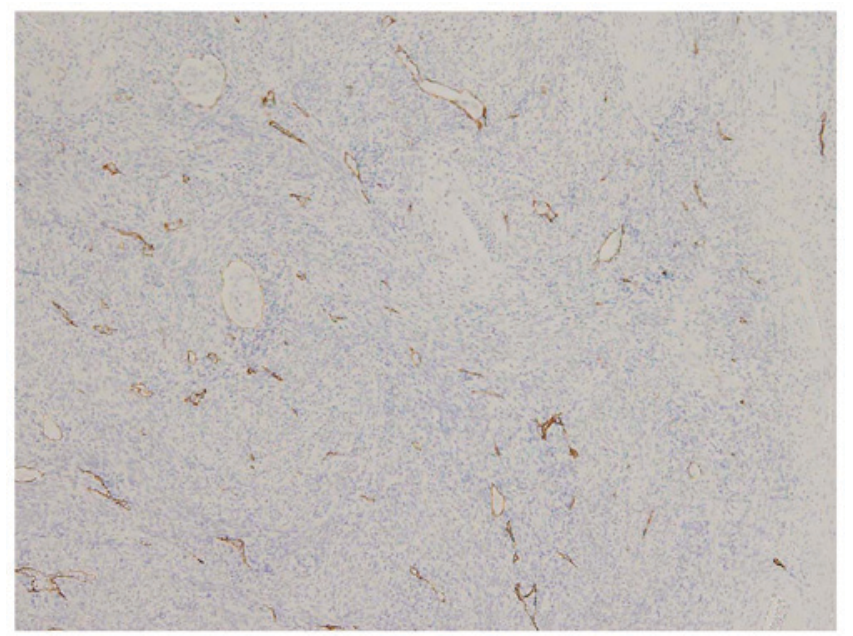

Figure 2. An actual view at 100 magnification $\left(2.3 \mathrm{~mm}^{2}\right)$ of an $\mathrm{LVI}^{+}$area in a peritumoral compartment. Lymphatic vessels with tumor invasion were present in a scattered way and counted as LVD with tumor invasion (magnification, x100, D2-40).

Spearman's rank correlation test was used to assess the correlation between the LVD with tumor invasion and the LVD in the $\mathrm{LVI}^{+}$areas of the peritumoral compartment of the $\mathrm{N}^{+}$group.

\section{Results}

On Hematoxylin and eosin (H\&E) slides, in the endometrium all cases showed continuous expansive proliferation of carcinoma with little stroma. In the myometrium, on the other hand, G1 and G2 tumors generally showed a discontinuous, adenomyosis-like pattern of infiltration with some desmoplastic stroma or muscular tissue intervening. In contrast, G3 tumors showed an expansive pattern of infiltration without intervening stroma as seen in the endometrium.

At least one LVI was suspected on H\&E slides and confirmed with D2-40 immunostaining in all 19 cases of the $\mathrm{N}^{+}$group. In the peritumoral compartment, the LVI was present (Fig. 2) in both the inner- and outer-half myometrium in 14 of the 19 cases, only in the inner-half in one case, in the outer-half in 3 cases and in neither of them in one case, which showed the LVI only in the intratumoral compartment (Table I). The number of cases available for examining the

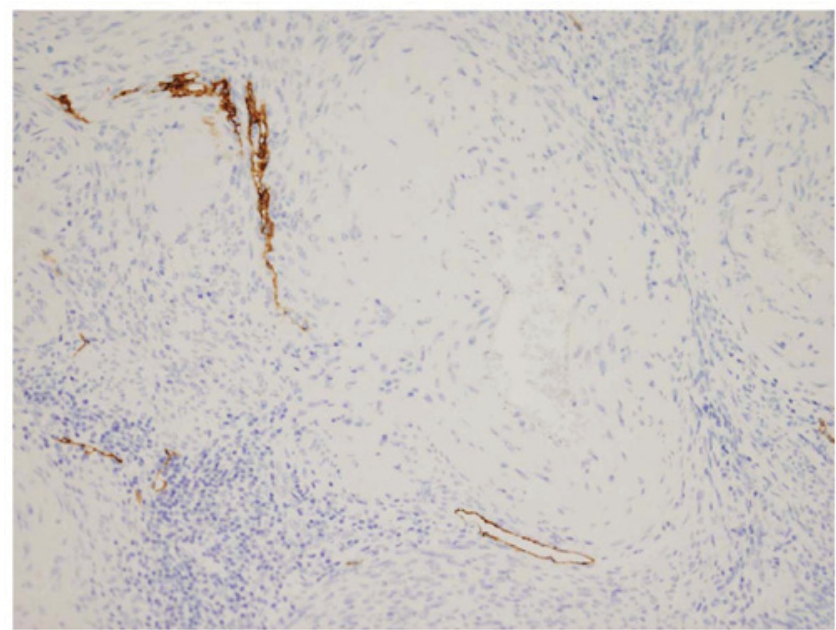

Figure 3. Close proximity between lymphatic vessels and arteries. Lymphatic vessels were present in a perivascular region (magnification, x200, D2-40).

LVD of the inner-half myometrium of the control compartment was 18 with one case showing ubiquitous LVI in the area excluded. A close proximity between the lymphatic vessels and small-to medium-sized arteries was noted in all compartments (Fig. 3).

In the $\mathrm{N}^{-}$group, even when LVI was unrecognized on $\mathrm{H} \& \mathrm{E}$ stained slides, at least one LVI was suspected on D2-40 immunostained slides in most of the cases. The absence of LVI was confirmed in only 2 cases.

$<$ LVDs of the $\mathrm{N}^{+}$and the $\mathrm{N}^{-}$groups $>$In the $\mathrm{N}^{+}$group (Table IIA) the LVDs of the $\mathrm{LVI}^{+}$and $\mathrm{LVI}^{-}$areas in the peritumoral and control compartmentswere $69.53 \pm 30.53$, $76.47 \pm 36.00$ and $34.17 \pm 26.02$ for the inner-half myometrium and $33.24 \pm 23.06,46.53 \pm 32.22$ and $16.37 \pm 8.38$ for the outer-half myometrium. The range of LVDs varied widely in both compartments. The LVDs with tumor invasion were $8.07 \pm 7.25$ and $6.94 \pm 9.98$ for the inner- and the outer-half myometrium, respectively. No correlation was found between the LVD with tumor invasion and LVD in both the inner- and outer-half myometrium of the peritumoral compartment (Spearman's rank correlation test).

In the $\mathrm{N}^{-}$group (Table IIB) the LVDs in the peritumoral and control compartments were $63.21 \pm 36.24$ and $46.10 \pm 59.38$ for the inner-half myometrium and $41.86 \pm 27.21$ and $18.21 \pm 9.53$ for the outer-half myometrium.

The comparison of the LVDs within each group is shown in Tables IIIA and IIIB. In the $\mathrm{N}^{+}$group (Table IIIA), the LVDs between the $\mathrm{LVI}^{+}$and the $\mathrm{LVI}^{-}$areas of both the innerand outer-half myometrium were not different $(\mathrm{P}=0.56$ for the inner-half myometrium and 0.17 for the outer-half myometrium. However, compared to the LVDs in the control compartment, those in the peritumoral compartment increased, irrespective of the presence of LVI ( $\mathrm{P}=0.0011$, 0.00025 for the $\mathrm{LVI}^{+}$and the $\mathrm{LVI}^{-}$areas of the inner-half myometrium, and 0.0099, 0.00079 for those of the outer-half myometrium). And the LVDs of the inner-half myometrium were higher than those of the outer-half myometrium in both the peritumoral and control compartments $(\mathrm{P}=0.00062$, $0.042,0.00016,0.010$ for the peritumoral compartment, and 0.012 for the control compartment). 
Table II. LVDs of $\mathrm{N}^{+}$and $\mathrm{N}^{-}$groups.

A, LVD of $\mathrm{N}^{+}$group

Mean \pm standard deviation (range)

\begin{tabular}{|c|c|c|c|c|c|c|}
\hline & \multicolumn{4}{|c|}{ Peritumoral compartment } & \multicolumn{2}{|c|}{ Control compartment } \\
\hline & \multicolumn{2}{|c|}{ Inner-half myometrium } & \multicolumn{2}{|c|}{ Outer-half myometrium } & \multirow{2}{*}{$\begin{array}{l}\text { Inner-half } \\
\text { myometrium } \\
(\mathrm{n}=18)\end{array}$} & \multirow{2}{*}{$\begin{array}{l}\text { Outer-half } \\
\text { myometrium } \\
(\mathrm{n}=19)\end{array}$} \\
\hline & $\begin{array}{c}\mathrm{LVI}^{+} \text {area } \\
(\mathrm{n}=15)\end{array}$ & $\begin{array}{l}\mathrm{LVI}^{-} \text {area } \\
(\mathrm{n}=19)\end{array}$ & $\begin{array}{c}\mathrm{LVI}^{+} \text {area } \\
(\mathrm{n}=17)\end{array}$ & $\begin{array}{l}\mathrm{LVI}^{-} \text {area } \\
(\mathrm{n}=19)\end{array}$ & & \\
\hline LVD & $\begin{array}{c}69.53 \pm 30.53 \\
(11-111)\end{array}$ & $\begin{array}{c}76.47 \pm 35.97 \\
(23-164)\end{array}$ & $\begin{array}{c}33.24 \pm 23.06 \\
(6-99)\end{array}$ & $\begin{array}{c}46.53 \pm 32.22 \\
(6-145)\end{array}$ & $\begin{array}{c}34.17 \pm 26.02 \\
\quad(0-101)\end{array}$ & $\begin{array}{c}16.37 \pm 8.38 \\
(3-33)\end{array}$ \\
\hline $\begin{array}{l}\text { LVD with tumor } \\
\text { invasion }{ }^{\mathrm{a}}\end{array}$ & $\begin{array}{c}8.07 \pm 7.25 \\
(2-22)\end{array}$ & Not applicable & $\begin{array}{c}6.94 \pm 9.98 \\
(1-43)\end{array}$ & Not applicable & Not applicable & Not applicable \\
\hline
\end{tabular}

$\mathrm{B}, \mathrm{LVD}$ of $\mathrm{N}^{-}$group

Mean \pm standard deviation (range)

\begin{tabular}{|c|c|c|c|}
\hline \multicolumn{2}{|c|}{ Peritumoral compartment } & \multicolumn{2}{|c|}{ Control compartment } \\
\hline Inner half myometrium & Outer half myometrium & Inner half myometrium & Outer half myometrium \\
\hline $\begin{array}{c}63.21 \pm 36.24 \\
(15-139)\end{array}$ & $\begin{array}{c}41.86 \pm 27.21 \\
(0-91)\end{array}$ & $\begin{array}{c}46.10 \pm 59.38 \\
(0-295)\end{array}$ & $\begin{array}{c}18.21 \pm 9.53 \\
(3-40)\end{array}$ \\
\hline
\end{tabular}

aThere is no correlation between the lymphatic vessel density (LVD) and LVD with tumor invasion (Spearman's rank correlation test).

In the $\mathrm{N}^{-}$group, as in the result of the $\mathrm{N}^{+}$group, LVDs of both inner- and outer-half myometrium of the peritumoral compartment were higher than those of the control compartment $(\mathrm{P}=0.0075$ and 0.00014$)$. Also, LVDs of the inner-half myometrium were higher than those of the outer-half myometrium in both the peritumoral and control compartments $(\mathrm{P}=0.021$ and 0.032$)$.

In both the $\mathrm{N}^{+}$and the $\mathrm{N}^{-}$groups, the differences in LVD between the peritumoral and control compartments were more statistically significant than those between the inner- and outer-half myometrium.

The differences in LVD of the peritumoral compartments between the groups were not significant $(\mathrm{P}=0.067$ and 0.29 for inner- and outer-half myometrium).

In the intratumoral compartment of the $\mathrm{N}^{+}$group, no lymphatic vessels were found in 16 of the 19 cases, and only 3 cases showed the presence of lymphatic vessels, 2 in the desmin-negative stroma and one in the solidly proliferating area of G3 tumor. All the 3 cases showed the LVI (Fig. 4). The ratio of the LVD with tumor invasion to the LVD were 3 to 18 (G2), 3 to 11 (G3) and 1 to 1 (G1) in the 3 cases (Table IV).

\section{Discussion}

In our study, twenty-four of the 202 cases (12\%) had nodal metastases. The rate was consistent with the relatively low incidence reported so far, which ranged from 10 to $15 \%$ (11).
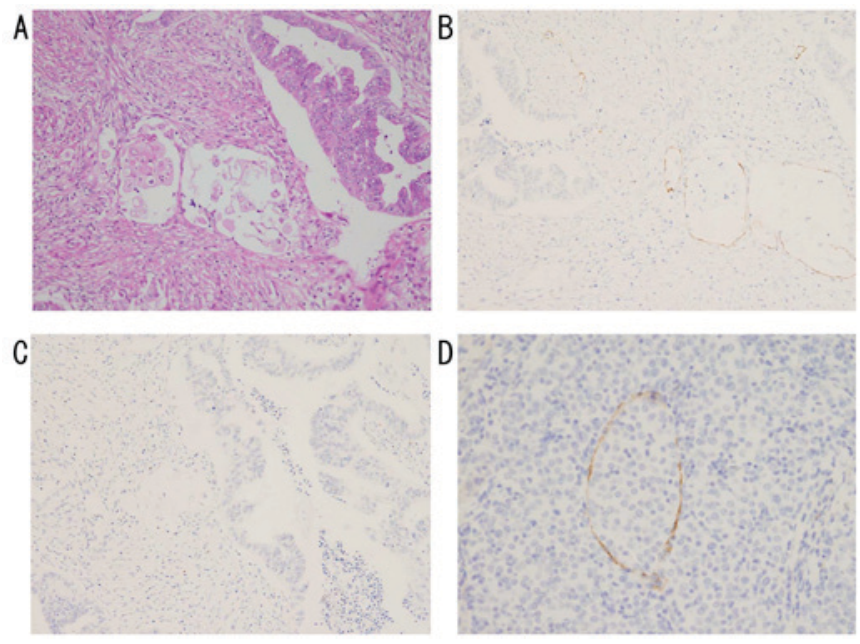

Figure 4. Lymphatic vessel invasion (LVI) of an intratumoral compartment. LVI was present in desmin-negative stroma (A) H\&E, (B) D2-40, (C) desmin, x200 magnification and in solid tumor (D) D2-40, x400 magnification.

The presence of LVI has been reported to be associated with nodal metastases in one article (11). In our study, the LVI was recognized in all 19 cases of the $\mathrm{N}^{+}$group with D2-40 immunostaining, and even in the $\mathrm{N}^{-}$group, the absence of LVI was confirmed only in 2 of 29 cases. Therefore, not the presence of LVI itself but the degree of LVI could be a possible predictor of nodal metastases. There was no correlation between 
Table III. Comparison of LVDs within each group.

A, Statistically significant differences in LVD in $\mathrm{N}^{+}$group

\begin{tabular}{|c|c|c|c|}
\hline \multicolumn{2}{|c|}{ Location } & \multirow[b]{2}{*}{ P-value } & \multirow[b]{2}{*}{ Statistical test } \\
\hline Peritumoral compartment & Control compartment & & \\
\hline \multicolumn{4}{|l|}{ Inner-half myometrium } \\
\hline $\mathrm{LVI}^{+}$ & & 0.0011 & Student's t-test \\
\hline $\mathrm{LVI}^{-}$ & & 0.00025 & Student's t-test \\
\hline \multicolumn{4}{|l|}{ Outer-half myometrium } \\
\hline $\mathrm{LVI}^{+}$ & & 0.0099 & Welch's test \\
\hline $\mathrm{LVI}^{-}$ & & 0.00079 & Welch's test \\
\hline Inner-half myometrium & Outer-half myometrium & & \\
\hline \multicolumn{4}{|l|}{ Peritumoral compartment } \\
\hline \multirow[t]{2}{*}{$\mathrm{LVI}^{+}$} & $\mathrm{LVI}^{+}$ & 0.00062 & Student's t test \\
\hline & $\mathrm{LVI}^{-}$ & 0.042 & Student's t test \\
\hline \multirow[t]{2}{*}{$\mathrm{LVI}^{-}$} & $\mathrm{LVI}^{+}$ & 0.00016 & Student's t test \\
\hline & $\mathrm{LVI}^{-}$ & 0.010 & Student's t test \\
\hline \multicolumn{4}{|l|}{ Control compartment } \\
\hline & & 0.012 & Welch's test \\
\hline
\end{tabular}

$\mathrm{B}$, Statistically significant differences in LVD in $\mathrm{N}^{-}$group

\begin{tabular}{|c|c|c|c|}
\hline \multicolumn{2}{|c|}{ Location } & \multirow[b]{2}{*}{ P-value } & \multirow[b]{2}{*}{ Statistical test } \\
\hline Peritumoral compartment & Control compartment & & \\
\hline Inner-half myometrium & & 0.0075 & Mann-Whitney's test \\
\hline Outer-half myometrium & & 0.00014 & Mann-Whitney's test \\
\hline Inner-half myometrium & Outer-half myometrium & & \\
\hline Peritumoral compartment & & 0.021 & Mann-Whitney's test \\
\hline Control compartment & & 0.032 & Mann-Whitney's test \\
\hline
\end{tabular}

$\mathrm{P}<0.05$ was considered statistically significant. LVD, lymphatic vessel density; LVI, lymphatic vessel invasion.

Table IV. LVD and LVD with tumor invasion in the intratumoral compartment in $\mathrm{N}^{+}$group.

\begin{tabular}{lcc}
\hline Cases & LVD & LVD with tumor invasion \\
\hline 16 cases & 0 & Not applicable \\
\hline 3 cases & & \\
Case 1 (G2) & 18 in stroma & 3 \\
Case 2 (G3) & 11 in tumor & 3 \\
Case 3 (G1) & 1 in stroma & 1 \\
\hline
\end{tabular}

LVD, lymphatic vessel density.

the LVD and frequency of LVI, and the LVD did not differ with the presence of LVI in the $\mathrm{N}^{+}$group. However, tumor lymphangiogesis was suggested, in that the LVDs in both the inner- and outer-half myometrium of the peritumoral compartments were higher than those of the control compartments. In both the $\mathrm{N}^{+}$and $\mathrm{N}^{-}$groups. Also, there was no difference in LVD of peritumoral compartments between the groups. Therefore, although tumor lymphangiogenesis increased LVD, the increased LVD was not related to the LVI and nodal metastases.

One of the factors to determine the LVD other than tumor lymphangiogenesis was the location in the myometrium. Not only in the peritumoral compartment but also in the control compartment, the LVDs in the inner-half myometrium were higher than those in the outer-half myometrium in both groups. Lymphatic vessels in uterine corpus showed an intimate association with arteries (Fig. 3) (13). Anatomically, in the inner third of the myometrium, abundant radial vessels from the subserosal arteries, the arcuate arteries, branched 
into the endometrium (12). Therefore, the richer vasculature in the inner-half myometrium should be one explanation for the higher LVD. This tendency was retained in the carcinoma-harboring uterine corpus regardless of the presence of LVI and nodal metastases.

Another influence on the LVD was the existing myometrium. There were no lymphatic vessels where the existing muscular tissue disappeared in 16 of 19 cases of the $\mathrm{N}^{+}$group. The result was in contrast to the high intratumoral LVD of gastric cancer, which generally had a high incidence of nodal metastases (17). Tumor lymphangiogenesis was also suggested, in that the remaining 3 cases showing lymphatic vessels also presented LVI, though the frequency (3/19) was low. In conclusion, the range of LVD varied widely in the uterine corpus. Our result showed that tumor lymphangiogenesis did not have an absolute impact on the LVD, LVI and nodal metastases in the carcinoma. The location, inner- or outer-half, in the myometrium, and the presence of the existing myometrium were important influences on the LVD other than tumor lymphangiogesis. The prognostic significance of the increased LVD caused by tumor lymphangiogenesis in the peritumoral compartment was not evident in endometrioid carcinoma of the uterine corpus in our study.

\section{References}

1. Franchi A, Gallo O, Massi D, Baroni G and Santucci M: Tumor lymphangiogenesis in head and neck squamous cell carcinoma: A morphometric study with clinical correlations. Cancer 101: 973-978, 2004.

2. Dadras SS, Paul T, Bertoncini J, Brown LF, Muzikansky A, Jackson DG, Ellwanger U, Garbe C, Mihm MC and Detmar M: Tumor lymphangiogenesis: A novel prognostic indicator for cutaneous melanoma metastasis and survival. Am J Pathol 162: 1951-1960, 2003.

3. Dadras SS, Lange-Asschenfeldt B, Velasco P, Nguyen L, Vora A, Muzikansky A, Jahnke K, Hauschild A, Hirakawa S, Mihm MC and Detmar M: Tumor lymphangiogenesis predicts melanoma metastasis to sentinel lymph nodes. Mod Pathol 18: 1232-1242, 2005.

4. Schoppmann SF, Bayer G, Aumayr K, Taucher S, Geleff S, Rudas M, Kubista E, Hausmaninger $H$, Samonigg $H$ Gnant M, et al: Prognostic value of lymphangiogenesis and lymphovascular invasion in invasive breast cancer. Ann Surg 240 306-312, 2004.
5. Li S and Li Q: Cancer stem cells, lymphangiogenesis, and lymphatic metastasis. Cancer Lett 357: 438-447, 2015.

6. Lohela M, Bry M, Tammela T and Alitalo K: VEGFs and receptors involved in angiogenesis versus lymphangiogenesis. Curr Opin Cell Biol 21: 154-165, 2009.

7. Raica M and Ribatti D: Targeting tumor lymphangiogenesis: An update. Curr Med Chem 17: 698-708, 2010.

8. Mannelqvist M, Stefansson I, Salvesen HB and Akslen LA: Importance of tumour cell invasion in blood and lymphatic vasculature among patients with endometrial carcinoma. Histopathology 54: 174-183, 2009.

9. Hachisuga T, Kaku T, Fukuda K, Eguchi F, Emoto M, Kamura T, Iwasaka T, Kawarabayashi T, Sugimori $\mathrm{H}$ and Mori M: The grading of lymphovascular space invasion in endometrial carcinoma. Cancer 86: 2090-2097, 1999.

10. Inoue Y, Obata K, Abe K, Ohmura G, Doh K, Yoshioka T, Hoshiai $\mathrm{H}$ and Noda K: The prognostic significance of vascular invasion by endometrial carcinoma. Cancer 78: 1447-1451, 1996.

11. Miyakuni Y, Matsumoto T, Arakawa A, Sonoue H, Suzuki C and Takeda S: Lymphatic invasion according to D2-40 immunostaining is a predictor of nodal metastasis in endometrioid adenocarcinoma of the uterine corpus. Pathol Int 58: 471-476, 2008.

12. Hendrickson RM, Atkins AK and Kempson LR: Uterus and Fallopian tubes. In: Histology for Pathologist. Mills ES (ed). 3rd edition. Lippincott Williams \& Wilkins, Philadelphia, PA, pp1016-1017, 2007.

13. Rogers PA, Donoghue JF and Girling JE: Endometrial lymphangiogensis. Placenta 29 (Suppl A): S48-S54, 2008.

14. Williams CS, Leek RD, Robson AM, Banerji S, Prevo R, Harris AL and Jackson DG: Absence of lymphangiogenesis and intratumoural lymph vessels in human metastatic breast cancer. J Pathol 200: 195-206, 2003.

15. Vleugel MM, Bos R, van der Groep P, Greijer AE, Shvarts A, Stel HV, van der Wall E and van Diest PJ: Lack of lymphangiogenesis during breast carcinogenesis. J Clin Pathol 57: 746-751, 2004.

16. Roma AA, Magi-Galluzzi C, Kral MA, Jin TT, Klein EA and Zhou M: Peritumoral lymphatic invasion is associated with regional lymph node metastases in prostate adenocarcinoma. Mod Pathol 19: 392-398, 2006.

17. Donizy P, Rudno-Rudzinska J, Halon A, Dziegala M, Kabarowski J, Frejlich E, Dziegiel P, Kielan W and Matkowski R: Intratumoral but not peritumoral lymphatic vessel density measured by D2-40 expression predicts poor outcome in gastric cancer-ROC curve analysis to find cut-off point. Anticancer Res 34: 3113-3118, 2014. 